\title{
Linking Only Aedes aegypti with Zika Virus Has World-Wide Public Health Implications
}

\author{
Fiona F. Hunter* \\ Centre for Vector-borne Diseases, Department of Biological Sciences, Brock University, St. Catharines, ON, Canada
}

Keywords: Zika virus (ZIKV), mosquito surveillance, vector competence, Aedes aegypti, Culex quinquefasciatus, Flaviviridae evolution, phylogenetics

\section{ZIKV MOSQUITO VECTORS ARE NOT WELL-ESTABLISHED}

Zika virus (ZIKV) is an emerging arbovirus in the Americans and as such, we know far less about the mosquito species involved in transmission than many experts, public health authorities, and politicians would have the public believe.

The entomological literature with respect to ZIKV and the supposedly pivotal role that Aedes aegypti plays in its transmission is frighteningly scant. Multiple mosquito species have tested positive for the virus in field-collected specimens, including 20 species from genus Aedes (in alphabetical order, A. aegypti, Aedes africanus, Aedes apicoargenteus, Aedes dalzieli, Aedes dorsalis, Aedes flavicollis, Aedes fowleri, Aedes furcifer, Aedes hirsutus, Aedes jamoti, Aedes luteocephalus, Aedes metallicus, Aedes minutus, Aedes neoafricanus, Aedes opok, Aedes taeniarostris, Aedes tarsalis, Aedes taylori, Aedes unilineatus, Aedes vittatus), as well as six non-Aedes species (Anopheles coustani, Anopheles gambiae, Mansonia uniformis, Eratmapodites inornatus, Eratmapodites quinquevittatus, and Culex perfuscus) (McCrae and Kirya, 1982; Haddow et al., 2012; Diallo et al., 2014).

In Malaysia, 58 pools of 1,277 A. aegypti, 59 pools of 4,492 Aedes albopictus, and 179 pools of 27,636 mosquitoes from 23 other Aedes species were surveyed for ZIKV and only a single pool of $A$. aegypti tested positive (Marchette et al., 1969). This was the first isolation of ZIKV outside of Africa (strain P6-740). In Gabon, 137 pools of 2,701 A. albopictus, 45 pools of 881 A. aegypti, 15 pools of 88 Aedes simpsoni complex, 29 pools of 690 Culex quinquefasciatus, and 21 pools of another 305 mosquitoes (made up of An. gambiae, Mansonia africana, M. uniformis, Culex sp., and E. quinquevittatus) were surveyed and two A. albopictus pools tested positive for ZIKV (Grard et al., 2014).

From the outset of the ZIKV outbreak in Brazil, the World Health Organization and other authorities have stated that $A$. aegypti is the mosquito that needs to be targeted for control in order to avoid a ZIKV pandemic; sometimes, A. albopictus is also included in the warnings. Novel strategies for controlling A. aegypti using Wolbachia-infected males or Oxitec ${ }^{\circledR}$ females (Yakob and Walker, 2016) target only A. aegypti. If ZIKV transmission is not being driven by A. aegypti, then these strategies will fail to protect people from ZIKV.

In mosquito surveys that followed the ZIKV outbreak on Yap Island in the Federated States of Micronesia, several species of mosquitoes were collected and tested for ZIKV; none was positive. This included a single negative A. aegypti specimen as well as 362 Aedes hensilli, the most abundant species, and 247 C. quinquefasciatus, the second most abundant (Ledermann et al., 2014). Subsequently, A. hensilli was experimentally infected with ZIKV MR766 (the original African lineage strain) and, despite relatively low dissemination rates, it was assumed that it served as a vector (Ledermann et al., 2014). 
However, Guerbois et al. (2016) were able to collect mosquitoes during the 2015 ZIKV outbreak in Chiapas State, Mexico. They tested 471 female A. aegypti mosquitoes in 55 pools of which 15 were positive for ZIKV by RT-PCR. The second most abundant species was $C$. quinquefasciatus but no pools tested positive for ZIKV. Unfortunately, Guerbois et al. (2016) were only able to isolate virus from 3 of the 15 RT-PCR-positive samples following inoculation onto VERO cells.

Taken together, these data suggest a role for A. aegypti in the transmission of ZIKV but the data also leave room for other mosquito species (and other non-vector modes of transmission) to contribute to ZIKV outbreaks.

\section{TRANSMISSION STUDIES INVOLVING A. AEGYPTI AND A. ALBOPICTUS}

In the 1950's $A$. aegypti mosquitoes were fed a blood-meal containing a ZIKV dose of $10^{6.7}$ mouse LD50 per $0.03 \mathrm{~mL}$. There was one successful ZIKV transmission after a group of three infected mosquitoes fed on a rhesus monkey. The authors concluded "until it can be shown that A. aegypti can be infected with lower virus doses than those used here, its efficiency as a vector of Zika virus under natural conditions remains uncertain" (Boorman and Porterfield, 1956).

In another study, A. aegypti mosquitoes were infected intrathoracically with ZIKV ArD 24280 (an African lineage ZIKV strain isolated from A. luteocephalus in 1976) and subsequently the mosquitoes transmitted ZIKV to suckling mice (Cornet et al., 1979). However, for transmission to occur naturally, virus must be ingested during a blood-meal, cross over the midgut epithelium into the hemolymph, disseminate throughout the body, and eventually cross the salivary gland epithelium into the gland's lumen. Then, as a mosquito feeds on a subsequent blood-meal host, she spits saliva (containing virus along with anticoagulants, vasodilators, and salivary peptides) into the host. The experiment with ArD 24280 demonstrated that there was no salivary gland barrier, but it shed no light on the issue of a potential midgut barrier; thus, the study did not provide evidence that A. aegypti is a competent vector in the wild. However, the study did compare the ability of A. aegypti to transmit YF vs. ZIKV (both via intrathoracic infection) and found that the incubation period for ZIKV was less than for YF. This would suggest that $A$. aegypti is likely an efficient ZIKV vector.

A research group from Singapore infected $A$. aegypti using a blood-meal with ZIKV MR766 at an initial infectious dose of $7.0 \log ^{10}$ tissue culture infectious dose $50\left(\mathrm{TCID}_{50} / \mathrm{mL}\right)$. They observed both high infection rates and high salivary gland dissemination rates (Li et al., 2012). Unfortunately, saliva was neither collected nor tested so conclusions about transmission could not be made. The same group then conducted very similar experiments with $A$. albopictus but this time they collected saliva and tested it for virus. They found that on day 10 postinfection $100 \%$ of mosquitoes $(n=12)$ had ZIKV in their saliva (Wong et al., 2013). These studies provide support for the assumption that $A$. albopictus (and probably $A$. aegypti) are good vectors of ZIKV MR766. Nevertheless, the infection, dissemination and transmission rates reported in the two studies are exceptionally high compared to what other researchers have found. Although mosquito strain differences and/or viral strain differences might cause these differences, it is possible that mosquito husbandry techniques also may have played a role. During extrinsic incubation, the mosquitoes were fed " $10 \%$ sugar/vitamin B complex ad libitum." Most (if not all) mosquito transmission studies use only an ad libitum sugar meal (although concentrations may vary) for daily maintenance of mosquitoes. It is possible that the addition of vitamin B complex to the sugar meal may be partially responsible for the high infection, dissemination, and transmission rates.

Four Aedes spp. from Senegal-A. aegypti, A. unilineatus, A. vittatus, and A. luteocephalus-were tested for their potential to transmit African lineage ZIKV isolates (MR766 and HD78788) in the lab. All four species were infected orally. ZIKV-positive saliva was only detected from A. vittatus and A. luteocephalus. Despite relatively high infection rates in $A$. aegypti, dissemination rates were low $(6.3 \%$ of 111 and $5.6 \%$ of 216 specimens from two different populations) and subsequent transmission rates (i.e., virus in saliva) were zero (Diagne et al., 2015).

Chouin-Carneiro et al. (2016) looked at the susceptibilities of A. aegypti and A. albopictus to an Asian lineage ZIKV strain (NC-2014-5132), fed at $10^{7} \mathrm{TCID}_{50} / \mathrm{mL}$. Similar to the Senegalese study (Diagne et al., 2015), authors found high infection rates but low dissemination and transmission rates for both species. Calculated transmission efficiencies were $3.3 \pm 3.3 \%$ for $A$. albopictus and $10 \pm 5.5 \%$ for A. aegypti. The authors concluded that "Ae. aegypti and Ae. albopictus were unexpectedly low competent vectors for ZIKV” (Chouin-Carneiro et al., 2016).

Aliota et al. (2016) studied the vector competence of $A$. aegypti. A. albopictus, Aedes triseriatus, and Culex pipiens fed on mice that had been infected with an Asian strain of ZIKV (PRVABC59). After 14 days' incubation, neither A. triseriatus nor $C$. pipiens had a disseminated infection or yielded virus in saliva. As positive controls, the researchers used A. aegypti and $A$. albopictus. They were able to detect virus in the saliva in 4 of 17 (24\%) A. aegypti fed $6.83 \log _{10} \mathrm{PFU} / \mathrm{mL}$ and in 2 of 9 (22\%) A. albopictus fed $6.02 \log _{10} \mathrm{PFU} / \mathrm{mL}$.

In a recent study, Jupille et al. (2016) tested the vector competence of two populations of $A$. aegypti (from the island of Madeira) and two populations of A. albopictus (from France) using a ZIKV strain from New Caledonia (NC-2014-5132). They concluded that neither species was very susceptible to ZIKV. Virus was detected in the saliva of 1 of 20 (5\%) A. aegpyti from Funchal and 0 of $20(0 \%)$ A. aegypti from Paul do Mar on day 9 post-infection; data were identical for $A$. aegpyti on day 14 . In contrast, the saliva of $A$. albopictus was negative for ZIKV for all specimens tested on day 9 from both Nice $(n=24)$ and Bar-surLoup $(n=24)$ and for A. albopictus tested on day 14 from Nice $(n=24)$. The saliva from only 1 of $24(4.2 \%)$ A. albopictus from Bar-sur-Loup was positive on day 14 .

Richard et al. (2016) tested the vector competence of two species implicated in 2013-2014 ZIKV outbreak in French Polynesia, using ZIKV strain PF13/251013-18. They found that "transmission efficiency was poor in A. aegypti" until 14 days post-infection and that $A$. polynesiensis was unable to transmit 
ZIKV at all. They concluded that there might be the "possible contribution of another vector for the propagation of ZIKV during the outbreak."

Ayres (2016) cautioned about placing too much emphasis on A. aegypti in the battle against ZIKV, and suggested that C. quinquefasciatus might be an important vector in Recife, Brazil. Huang et al. (2016) looked at the vector competence of colonized C. pipiens and C. quinquefasciatus but were unable to demonstrate infection or dissemination at 7 or 14 days postinfection in mosquitoes held at $28^{\circ} \mathrm{C}$. Fernandes et al. (2016) were unable to show transmission in C. quinquefasciatus.

The first published report of the vector competence of $C$. quinquefasciatus comes from Guo et al. (2016). They were able to demonstrate that mosquitoes held at $29^{\circ} \mathrm{C}$ could transmit ZIKV by bite to suckling mice and furthermore, that the peak time of virus appearance in the salivary glands was day 8 post-infection.

With all of the conflicting reports in the literature, it is prudent to consider other evidence that might shed light on which mosquito species are involved in ZIKV transmission.

\section{EVIDENCE FROM PHYLOGENETICS}

There is an additional line of evidence-the evolutionary history of the Flaviviridae-that points to species other than $A$. aegypti as playing key roles in the current ZIKV outbreaks. Numerous authors have reconstructed phylogenies of the Flaviviridae based on amino acid sequences and nucleotide sequences. Figure $\mathbf{1}$ is a synthesis of three papers (Kuno et al., 1998; Lanciotti et al., 2008; Moureau et al., 2015). According to the International Commission on Viral Taxonomy (ICVT), West Nile virus (WNV), and Saint Louis Encephalitis virus (SLE) are designated as "Culex-associated" viruses whereas Dengue virus (DENV), Yellow Fever virus (YF), and ZIKV are "Aedes-associated" viruses (see also Moureau et al., 2015). The "Culex-associated" flaviviruses are known for their bird reservoirs and human neurotropic effects (e.g., encephalitis and paralysis) whereas "Aedes-associated" flaviviruses such as Dengue virus (DENV) and Yellow Fever virus (YF) are known for primate reservoirs and hemorrhagic diseases.

Phylogenetically, ZIKV clearly belongs to the lineage that contains WNV and SLE, two so-called "Culex-associated" viruses. Nodal support for the [ZIKV $+[\mathrm{SLE}+\mathrm{WNV}]]$ clade ranges from 97 to 100\% (Kuno et al., 1998; Lanciotti et al., 2008; Moureau et al., 2015). However, several of these same authors still consider ZIKV to be Aedes-associated according to convention. Based on the phylogenetic relationships, the most parsimonious interpretation is to include ZIKV within the Culex-associated lineage. This is in line with comments made by Grard et al. (2010) who analyzed the complete coding sequence of Aedes-borne flaviviruses [sensu ICVT] and concluded that, based on an analysis of amino acid distances in the NS5 gene, ZIKV is "clearly related to Culex-borne flaviviruses." Using this logic, the fact that $\mathrm{ZIKV}$ is linked to neurotropic effects-such as fetal brain

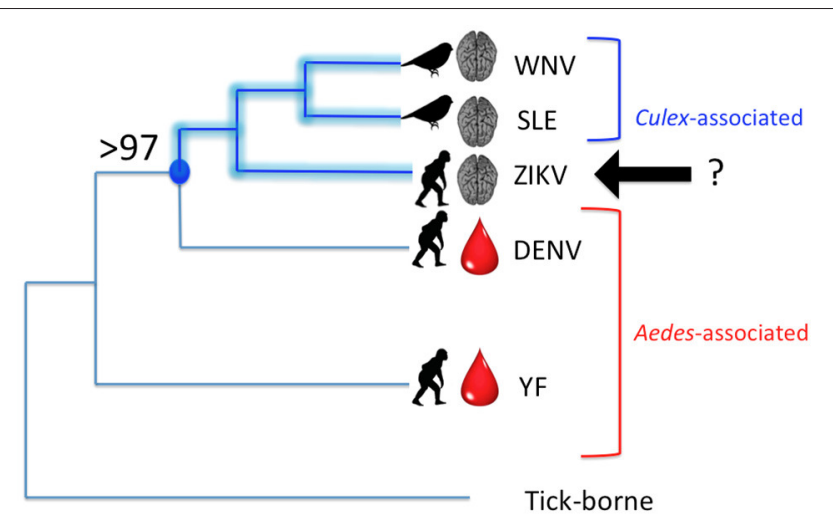

FIGURE 1 | Phylogenetic relationships among several mosquito-borne Flaviviruses. WNV, West Nile virus; SLE, Saint Louis Encephalitis virus; ZIKV, Zika virus; DENV, Dengue virus; YF, Yellow fever virus. Lengths of branches do not reflect phylogenetic distances, but tree topology is in agreement with several published phylogenies, including Moureau et al. (2015). Brackets indicate "Culex"-associated and "Aedes"-associated viruses, in keeping with the ICVT, but with ZIKV unassigned. A crucial node (indicated in blue) is supported by $97-100 \%$ of molecular analyses of the Flavivirus genome. This node defines the clade of [ZIKV + [SLE + WNV]]. Disease indicators are overlaid on the phylogeny with a brain representing neurotropic effects and a drop of blood, hemorrhagic effects. Known reservoir hosts are shown with either a bird silhouette or a primate silhouette.

abnormalities and Guillain-Barré syndrome-comes as no surprise. Due to the relatively low number of human ZIKV cases prior to 2007, severe neurotropic symptoms were previously unknown, and were unexpected due to the paradigm that ZIKV was a traditional "Aedes-borne" virus [sensu ICVT] akin to DENV.

Figure 1 also shows the association with disease indicators, namely neurotropic effects (indicated by a brain) or hemorrhagic effects (indicated by a drop of blood). The common vertebrate reservoirs are also indicated with silhouettes of either birds or non-human primates. In terms of disease indicators, ZIKV is more like WNV and SLE, but possibly more like DENV and YF in terms of reservoir hosts. Or is it? A forgotten paper by Okai et al. (1971) reported that 15\% of 221 birds collected in Uganda tested positive for ZIKV antibodies by Hemagglutination Inhibition Assays, with the majority of positive birds being Greenbuls (Family Pycnonotidae). The Okai et al. (1971) study would support the addition of a bird silhouette to the ZIKV branch in Figure 1.

\section{KEEP AN OPEN MIND}

Scientists need to consider the possibility that ZIKV may be more similar to the classic "Culex-associated" flaviviruses than it is to the "Aedes-associated" viruses by collecting and testing field-collected Culex spp., by screening a number of different vertebrates for their potential roles as reservoirs, and by studying the vector competence of Culex spp. in the laboratory. Aliota et al. (2016) have reported that colony-reared C. pipiens were unable to become infected with ZIKV strain 
PRVABC59. Weger-Lucarelli et al. (2016) also showed that colony-reared C. pipiens, C. quinquefasciatus, and C. tarsalis were refractory to ZIKV strain PRVABC59. However, additional studies on the vector competence of Culex mosquitoes to other ZIKV strains need to be conducted and published. In certain geographic regions, C. quinquefasciatus is a highly peridomestic mosquito and it feeds avidly on humans; furthermore, it has been found in relatively large numbers when mosquito surveillance has been conducted for ZIKV (Grard et al., 2014: Gabon, third most abundant species; Ledermann et al., 2014: Yap, second most abundant species). The World cannot afford to concentrate all of its efforts on monitoring and controlling A. aegypti in the fight against ZIKV, especially if there are Culex mosquitoes that may also serve as competent vectors.

Consideration ought to be given to the phylogenetic evidence that "Culex-borne" flaviviruses have evolved from

\section{REFERENCES}

Aliota, M. T., Peinado, S. A., Osorio, J. E., and Bartholomay, L. C. (2016). Culex pipiens and Aedes triseriatus mosquito susceptibility to Zika virus. Emerg. Infect. Dis. 22, 1857-1859. doi: 10.3201/eid2210.161082

Ayres, C. F. J. (2016). Identification of Zika virus vectors and implications for control. Lancet Infect. Dis. 16, 278-279. doi: 10.1016/S1473-3099(16)00 073-6

Boorman, J. P. T., and Porterfield, J. S. (1956). A simple technique for infection of mosquitoes with viruses: transmission of Zika virus. Trans. R. Soc. Trop. Med. Hyg. 50, 238-242. doi: 10.1016/0035-9203(56)90029-3

Chouin-Carneiro, T., Vega-Rua, A., Vazeille, M., Yebakima, A., Girod, R., Goindin, D., et al. (2016). Differential susceptibilities of Aedes aegypti and Aedes albopictus from the Americas to Zika virus. PLoS Negl. Trop. Dis. 10:e0004543. doi: 10.1371/journal.pntd.0004543

Cornet, M., Robin, Y., Adam, C., Valade, M., and Calvo, M. A. (1979). Transmission expérimentale comparée du virus amaril et du virus Zika chez Aedes aegypti L. Cah. ORSTOM Sér. Ent. Méd. Parasitol. 17, 47-53.

Diagne, C. T., Diallo, D., Faye, O., Ba, Y., Faye, O., Gaye, A., et al. (2015). Potential of selected Senegalese Aedes spp. mosquitoes (Diptera: Culicidae) to transmit Zika virus. BMC Infect. Dis. 15:492. doi: 10.1186/s12879-015-1 231-2

Diallo, D., Sall, A. A., Diagne, C. T., Faye, O., Faye, O., Ba, Y., et al. (2014). Zika virus emergence in mosquitoes in Southeastern Senegal, 2011. PLoS ONE 9:e109442. doi: 10.1371/journal.pone.0109442

Evans, M. V., Dallas, T. A., Han, B. A., Murdock, C. C., and Drake, J. M. (2017). Data-driven identification of potential Zika virus vectors. Elife 6:e22053. doi: 10.7554/elife.22053

Fernandes, R. S., Campos, S. S., Ferreira-de-Brito, A., Miranda, R. M., Barbosa da Silva, K., Castro, M. G. et al. (2016). Culex quinquefasciatus from Rio de Janeiro is not competent to transmit the local Zika virus. PLoS Negl. Trop. Dis. 10:e0004993. doi: 10.1371/journal.pntd.00 04993

Grard, G., Caron, M., Mombo, I. M., Nkoghe, D., Ondo, S. M., Jiolle, D., et al. (2014). Zika virus in Gabon (Central Africa) - 2007: a new threat from Aedes albopictus? PLoS Negl. Trop. Dis. 8:e2681. doi: 10.1371/journal.pntd.000 2681

Grard, G., Moureau, G., Charrel, R. N., Holmes, E. C., Gould, E. A., and de Lamballerie, X. (2010). Genomics and evolution of Aedes-borne flaviviruses. J. Gen. Virol. 91, 87-94. doi: 10.1099/vir.0.014506-0

Guerbois, M., Fernandez-Salas, I., Azar, S. R., Danis-Lozano, R., AlpucheAranda, C., et al. (2016). Outbreak of Zika virus infection, Chiapas State, Mexico, 2015, and first confirmed transmission by Aedes aegypti ancestral "Aedes-borne" flaviviruses (Grard et al., 2010) and that means that an expanded (rather than a restricted) vector range might be expected for ZIKV. Furthermore, based on a data-driven model linking mosquito vector species and vector-virus traits, Evans et al. (2017) have predicted that as many as 35 different mosquito species could be vectors for ZIKV.

Medical entomologists, public health professionals, and politicians are urged to keep an open mind on the issue of which mosquito species need to be targeted for control in the battle against ZIKV.

\section{AUTHOR CONTRIBUTIONS}

The author confirms being the sole contributor of this work and approved it for publication. mosquitoes in the Americas. J. Infect. Dis. 214, 1349-1356. doi: 10.1093/infdis/j iw302

Guo, X., Li, C., Deng, Y., Xing, D., Liu, Q., Wu, Q., et al. (2016). Culex pipiens quinquefasciatus: a potential vector to transmit Zika virus. Emerg. Microbes Infect. 5:e102. doi: 10.1038/emi.2016.102

Haddow, A. D., Schuh, A. J., Yasuda, C. Y., Kasper, M. R., Heang, V., Huy, R., et al. (2012). Genetic characterization of Zika virus strains: geographic expansion of the Asian lineage. PLoS Negl. Trop. Dis. 6:e1477. doi: 10.1371/journal.pntd.0001477

Huang, Y.-J., Ayers, V. B., Lyons, A., Unlu, I., Alto, B. W., and Cohnstaedt, L. W. (2016). Culex species mosquitoes and Zika virus. Vector Borne Zoonotic Dis. 16, 673-676. doi: 10.1089/vbz.2016 .2058

Jupille, H., Seixas, G., Mousson, L., Sousa, C. A., and Failloux, A.-B. (2016) Zika Virus, a new threat for Europe? PLoS Negl. Trop. Dis. 10:e0004901. doi: 10.1371/journal.pntd.0004901

Kuno, G., Chang, G. J., Tsuchiya, K. R., Karabatsos, N., and Cropp, C. B. (1998). Phylogeny of the genus Flavivirus. J. Virol. 72, 73-83.

Lanciotti, R. S., Kosoy, O. L., Laven, J. J., Velez, J. O., Lambert, A. J., Johnson, A J., et al. (2008). Genetic and serological properties of Zika virus associated with an epidemic, Yap State, Micronesia, (2007). Emerg. Infect. Dis. 14, 1232-1239. doi: 10.3201/eid1408.080287

Ledermann, J. P., Guillaumot, L., Yug, L., Saweyog, S. C., Tided, M., Machieng, P., et al. (2014). Aedes hensilli as a potential vector of Chikungunya and Zika viruses. PLoS Negl. Trop. Dis. 8:e3188. doi: 10.1371/journal.pntd.0003188

Li, M. I., Wong, P. S. J., Ng, L. C., and Tan, C. H. (2012). Oral susceptibility of Singapore Aedes (Stegomyia) aegypti (Linnaeus) to Zika virus. PLoS Negl. Trop. Dis. 6:e1792. doi: 10.1371/journal.pntd.0001792

Marchette, N. J., Garcia, R., and Rudnick, A. (1969). Isolation of Zika virus from Aedes aegypti mosquitoes in Malaysia. Am. J. Trop. Med. Hyg. 18, 411-415. doi: 10.4269/ajtmh.1969.18.411

McCrae, A. W., and Kirya, B. G. (1982). Yellow fever and Zika virus epizootics and enzootics in Uganda. Trans. R. Soc. Trop. Med. Hyg. 76, 552-562. doi: 10.1016/0035-9203(82)90161-4

Moureau, G., Cook, S., Lemey, P., Nougairede, A., Forrester, N. L., Khasnatinov, M., et al. (2015). New insights into Flavivirus evolution, taxonomy and biogeographic history, extended by analysis of canonical and alternative coding sequences. PLoS ONE 10:e0117849. doi: 10.1371/journal.pone.0117849

Okai, N. O., George, P. V., Tukei, P. M., Kafuko, G. W., and Lule, M. S. (1971). Arbovirus survey in wild birds in Uganda. East Afr. Med. J. 48, 725-731.

Richard, V., Paoaafaite, T., and Cao-Lormeau, V.-M. (2016). Vector competence of French Polynesian Aedes aegypti and Aedes polynesiensis for Zika virus. PLoS Negl. Trop. Dis. 10:e0005024. doi: 10.1371/journal.pntd.0005024 
Weger-Lucarelli, J., Rueckert, C., Chotiwan, N., Nguyen, C., Garcia Luna, S. M., Fauver, J. R., et al. (2016). Vector competence of American mosquitoes for three strains of Zika virus. PLoS Negl. Trop. Dis. 10:e0005101. doi: 10.1371/journal.pntd.0005101

Wong, P. J., Li, M. I., Chong, C., Ng, L., and Tan, C. (2013). Aedes (Stegomyia) albopictus (Skuse): a potential vector of Zika virus in Singapore. PLoS Negl. Trop. Dis. 7:e2348. doi: 10.1371/journal.pntd.0002348

Yakob, L., and Walker, T. (2016). Zika virus outbreak in the Americas: the need for novel mosquito control methods. Lancet Glob Health 4, e148-e149. doi: $10.1016 / \mathrm{s} 2214-109 \mathrm{x}(16) 00048-6$
Conflict of Interest Statement: The author declares that the research was conducted in the absence of any commercial or financial relationships that could be construed as a potential conflict of interest.

Copyright $\odot 2017$ Hunter. This is an open-access article distributed under the terms of the Creative Commons Attribution License (CC BY). The use, distribution or reproduction in other forums is permitted, provided the original author(s) or licensor are credited and that the original publication in this journal is cited, in accordance with accepted academic practice. No use, distribution or reproduction is permitted which does not comply with these terms. 